\title{
Drug Incorporation into Polymer Filament Using Simple Soaking Method for Tablet Preparation Using Fused Deposition Modeling
}

\author{
Tatsuaki Tagami, ${ }^{a}$ Eriko Kuwata, ${ }^{a}$ Norihito Sakai, ${ }^{b}$ and Tetsuya Ozeki*,a \\ ${ }^{a}$ Drug Delivery and Nano Pharmaceutics, Graduate School of Pharmaceutical Sciences, Nagoya City University; \\ 3-1 Tanabe-dori, Mizuho-ku, Nagoya 467-8603, Japan: and ${ }^{b}$ The Nippon Synthetic Chemical Industry Co., Ltd.; \\ 2-4 Komatsubara-cho, Kita-ku, Osaka 530-0018, Japan. \\ Received June 10, 2019; accepted July 12, 2019
}

The use of three-dimensional (3D) printing technology is expanding in various fields. The application of 3D printing is expected to increase in the pharmaceutical industry after 3D-printed tablets were approved by the U.S. Food and Drug Administration (FDA). Fused deposition modeling (FDM), a type of 3D printing, has been extensively studied for the manufacturing of tablets. A drug-loaded polymer filament, the ink of FDM 3D printers, can be prepared using the hot melt extrusion method or a simple drug-soaking method. In the present study, we investigate the influence of the experimental conditions on the loading of curcumin (model drug with fluorescence) into a polyvinylalcohol polymer filament using the soaking method. We show that organic solvent type (isopropanol, methanol, acetone, and ethanol), temperature ( 25 and $80^{\circ} \mathrm{C}$ ), and $\mathrm{drug}$ concentration $(2-333 \mathrm{mg} / \mathrm{mL})$ greatly affect drug loading. Around $5 \%$ curcumin can be incorporated into the polyvinylalcohol filament using the soaking method. The drug dissolution from 3D-printed tablets depends on the drug content in the polymer filament. The incorporation of a higher amount of curcumin, which has poor water solubility, greatly delays drug dissolution. These results provide useful information on the preparation of 3D-printed tablets using a drug-loaded polymer filament obtained with the soaking method.

Key words controlled release; drug loading; fused deposition modeling (FDM); polyvinylalcohol (PVA); tablet; three-dimensional (3D) printing

\section{INTRODUCTION}

The use of three-dimensional (3D) printing has been expanding in various fields, including aerospace, construction, food, education, and medicine. An increasing number of studies have been conducted on 3D-printed tablets, which stems from the U.S. Food and Drug Administration approval of 3Dprinted tablets (orodispersible tablets for epileptic patients) in August 2015. The use of 3D printing allows the manufacturing of high-dose orodispersible tablets, ${ }^{1,2)}$ whereas it is difficult to manufacture such tablets using a conventional method. The 3D printing of tablets and other drug formulations has received attention. Additionally, although 3D-printed tablets are currently manufactured in pharmaceutical laboratories, 3D printers are compact and may thus allow manufacturing at hospitals and pharmacies. Several groups have proposed local preparation for personalized medicine., ${ }^{3,4}$

Among the various types of 3D printers, ${ }^{2,5)}$ fused deposition modeling (FDM), which has a good cost/performance ratio, is commonly used in industry and for personal use. Many recently published studies utilized FDM for the preparation of 3D-printed tablets, which can be tailored for individual patients. For example, models for tailoring dosing through FDM have been reported. Tablets of various sizes containing warfarin $^{6)}$ and theophylline ${ }^{7)}$ were prepared using a 3D printer. Goyanes et al. investigated patients' acceptability of 3D-printed placebo tablets with different shapes and colors made using an FDM printer. ${ }^{8)}$ 3D-printed tablets with unique geometry have been investigated. 3D-printed tablets with different fill densities (parameters can be changed in software) can be used to control drug release ${ }^{9)}$ and made to float in the gastrointestinal tract. ${ }^{10,11)}$ A model of a polypill, which is a tablet that contains multiple active pharmaceutical ingredients for cardiovascular diseases, has been reported as a strategy for drug adherence. ${ }^{12}$ ) The preparation of various kinds of unique tablets using FDM printers has been extensively investigated for personalized therapy.

FDM printers use a polymer filament as the printer ink. The polymer filament is inserted into the nozzle and melted using a heating device. The melted polymer ink is extruded from the heated nozzle. The printing is a layer-by-layer process, called additive manufacturing, that starts from the bottom of the designed object. Various compositions of drugloaded polymer filament have been investigated, such as methacrylic polymers (Eudragit series), cellulose-based polymers (hydroxypropyl celluloses), a vinylpyrrolidone-based polymer (Kollidone), and polyvinylalcohol (PVA), as printer inks. ${ }^{6-13)}$ PVA has been used to support 3D-printed objects because it is easy to wash away with water. The 3D printing conditions for PVA filaments and other conventional plastic materials such as polylactic acid and acrylonitrile butadiene styrene have been well investigated. The water solubility and pharmaceutical utility of PVA make it a promising base material for 3Dprinted tablets.

Drug-loaded polymer filaments for FDM printing have been prepared using two methods. ${ }^{14)}$ Hot melt extrusion (HME) is a process that blends a thermoplastic polymer and a drug at elevated temperature. HME has been used to mask drug bitterness, improve drug dissolution, and enable sustained drug release. ${ }^{15)}$ As HME is applicable for the preparation of polymer filaments for FDM printing, the preparation of polymer filaments for 3D printing has been reported. ${ }^{16)}$ Drug-loaded 
polymer filaments for FDM printing can also be prepared by simply soaking the polymer filament in a drug solution. This simple method uses a compound that is a non-solvent for the polymer filament (e.g., ethanol for a PVA filament) during soaking and a solvent for the drug (i.e., ethanol-soluble drug in this case). The soaking method for 3D printing has been reported among several groups. ${ }^{14)}$ The method was used to incorporate several drugs into a polymer in ethanolic solution for an appropriate time (e.g., 24h). It was found that the drug loading amount can be varied. The soaking method is simple and does not require heating, unlike HME. Although the drug loading efficiency is generally lower than that for HME, the soaking method may be applicable for highly potent drugs, for which large amounts are not required. However, it is unclear how the conditions of the soaking method affect drug loading efficiency. In the present study, we investigate the effects of the soaking method conditions on loading into a PVA filament. We found that the organic solvent type, temperature, and drug concentration affect drug loading and the drug dissolution profile.

\section{MATERIALS AND METHODS}

Reagents Curcumin extract (from Curcuma longa) was purchased from Sigma-Aldrich (St. Louis, MO, U.S.A.). Synthetic curcumin was purchased from Tokyo Chemical Industry Co., Ltd. (Tokyo, Japan). PVA filament was obtained from Nippon Synthetic Chemical Industry Co., Ltd. (Osaka, Japan). Isopropanol (IPA), methanol $(\mathrm{MeOH})$, acetone (ACE), ethanol $(\mathrm{EtOH})$, and Tween 20 were purchased from FUJIFILM Wako Pure Chemical Corporation (Osaka, Japan).

Preparation of Curcumin-Loaded PVA Filament In a typical experiment, approximately $10 \mathrm{~cm}$ of PVA filament cut using micro nippers was placed in a $15-\mathrm{mL}$ disposable conical tube and $15 \mathrm{~mL}$ of curcumin-dispersed organic solvent was added into the tube. For the experiments of scaling up, coiled PVA filament of an appropriate length $(1 \mathrm{~m})$ was soaked with curcumin dispersion $(150 \mathrm{~mL}$ of ethanol) in an appropriate screw-top glass container. The lid of the tube was screwed on tightly and the sample was incubated at 25 or $80^{\circ} \mathrm{C}$ in a water bath. After incubation, the filament was collected from the tube. For the tube of samples incubated at $80^{\circ} \mathrm{C}$, the lid was difficult to remove and thus opened after cooling to prevent the bumping of samples. The drug-loaded PVA filament was gently rinsed with an organic solvent and dried at $40^{\circ} \mathrm{C}$ in an incubator that contained silica gel overnight.

Determination of Curcumin Content in PVA Filament and Solution The curcumin-loaded PVA filament was cut into fragments using micro nippers. The two ends of the filament were excluded from measurements. The fragments were weighed using an electrical balance (approximately $15-30 \mathrm{mg}$ ). A fragment and a magnetic stirrer were placed in a glass vial and $10 \mathrm{~mL}$ of $0.1 \%$ Tween 20 solution was added. The sample was stirred at room temperature for $1 \mathrm{~h}$ to dissolve it. For samples that were difficult to dissolve, the vials were warmed at $80^{\circ} \mathrm{C}$ in a water bath and the samples were stirred again. The warming and stirring process was repeated until the sample had dissolved completely. Then, the sample was diluted with $0.1 \%$ Tween 20 solution, and $200 \mu \mathrm{L}$ of the sample solution was transferred to a 96-well black plate. The fluorescence of curcumin was measured using a Wallac 1420 ARVO plate reader (PerkinElmer, Inc., Waltham, MA, U.S.A.) (ex: $485 \mathrm{~nm}$; em: $535 \mathrm{~nm})$.

For the determination of curcumin amount dissolved in the organic solvent, the solution was filtered through $0.45-\mu \mathrm{m}$ pores and diluted with $0.1 \%$ Tween 20 solution, and the fluorescence of curcumin was measured using a plate reader (PerkinElmer, Inc.). At least 100-fold dilution of curcumin solution dissolved with an organic solvent was conducted to prevent the influence of the organic solvent.

Powder X-Ray Diffraction The curcumin-loaded PVA filaments prepared in the previous section were cut into minute fragments using micro nippers. These PVA fragments were further pulverized using an electric coffee grinder. The pulverized samples and curcumin samples were loaded onto sample holders, and the samples were measured using X-ray diffraction (XRD; Rint-Ultima, Rigaku Co., Ltd., Tokyo, Japan) with $\mathrm{Cu}-\mathrm{K} \alpha$ radiation. The $\mathrm{X}$-ray tube voltage and current were set at $44 \mathrm{kV}$ and $40 \mathrm{~mA}$, respectively.

Differential Scanning Calorimetry The pulverized samples prepared in the previous section were also used for differential scanning calorimetry (DSC). The DSC curves were obtained using a DSC-60 differential scanning calorimeter (Shimadzu, Kyoto, Japan). Approximately 2-3 mg of a sample was mounted onto an aluminum pan, covered with a lid, and sealed with crimpers (Shimadzu). The heating rate and scanning temperature range were set to $10^{\circ} \mathrm{C} / \mathrm{min}$ and 30 to $250^{\circ} \mathrm{C}$, respectively.

Preparation of 3D-Printed Tablets The 3D printing of tablets was conducted as described in our previous study. ${ }^{10,17)}$ Briefly, a cylindrical object (diameter: $7.0 \mathrm{~mm}$; height: $3.5 \mathrm{~mm}$ ) was designed using 123D Design 3D computer-assisted design (CAD) software (Autodesk Inc., San Rafael, CA, U.S.A.). Then, the STL file created by the 3D CAD software was transferred to Cura slicer software (Ultimaker; Geldermalsen, the Netherlands), and the 3D printing program was set in the software. The 3D printing conditions were as follows: a layer height of $0.2 \mathrm{~mm}$, a printing speed of $20 \mathrm{~mm} / \mathrm{s}$, a printing temperature of $210^{\circ} \mathrm{C}$, a bed temperature of $60^{\circ} \mathrm{C}$, and a flow of $120 \%$. The g-code file converted by the slicer software was executed using 3D printing host software (Pronterface; GNU General Public License) that controlled the FDM printer (FDM-200W, Ninjabot, Shizuoka, Japan). The curcuminloaded PVA filament prepared in the previous section was set into the nozzle of the $3 \mathrm{D}$ printer and tablets were printed.

Dissolution Test A dissolution test (paddle method) was conducted following the Japanese Pharmacopoeia 17th edition with modification. Briefly, the dissolution vessels were filled with $900 \mathrm{~mL}$ of $0.1 \%$ Tween 20 solution. The sample solution was maintained at $37^{\circ} \mathrm{C}$, and the stirring rate was maintained at $100 \mathrm{rpm}$ by the dissolution apparatus (NTR-6200 A; Toyama Sangyo, Osaka, Japan). The tablet samples were placed into dissolution sinkers. The samples were removed at the appropriate time point and $200 \mu \mathrm{L}$ of the sample solution was transferred to a 96-well black plate. The fluorescence of curcumin in the samples was measured using a Wallac 1420 ARVO plate reader (PerkinElmer, Inc.).

Kinetics of Drug Release The fitting of drug dissolution profile against kinetics models was analyzed by using Microsoft Excel program, as described in previous review article. $^{18,19)}$

Drug dissolution of zero-order model is represented by the 
Eq. (1). $Q$ is the amount of released drug in time $t . K_{0}$ is the zero order constant.

$$
Q=K_{0} t
$$

Drug dissolution of first-order model is represented by the Eq. (2). $Q_{1}$ is the amount of released drug in time $t . Q_{0}$ is the initial amount of drug. $K$ is the first order constant.

$$
\log Q_{1}=\log Q_{0}-K t / 2.303
$$

Drug dissolution of Higuchi model is represented by the Eq. (3). $Q$ is the amount of released drug in time $t . D$ is drug diffusivity. $C$ and $C_{\mathrm{s}}$ are initial drug concentration of and drug solubility, respectively.

$$
Q=\sqrt{D\left(2 C-C_{\mathrm{s}}\right) C_{\mathrm{s}} t}
$$

Drug dissolution of Korsmeyer-Peppas model is represented by the Eq. (4). $M_{t} / M_{\infty}$ is release rate of drug in time $t$. The value of $M_{t} / M_{\infty}<0.6$ was applied for the analysis. According to cylindrical tablet, $\mathrm{n}$ value indicates following that: $0.45 \leq n$ means Fickian diffusion mechanism, $0.45<n<0.89$ means non-Fickian transport. $n=0.89$ means Case II transport, $0.89<n$ means super Case II transport.

$$
M_{t} / M_{\infty}=K t^{n}
$$

\section{RESULTS AND DISCUSSION}

Influence of Organic Solvent Type on Curcumin Loading into PVA Filament We investigated drug loading into a PVA filament using the soaking method. There is little information on drug loading using this method as no systematic studies have been conducted. Curcumin, which has antioxidant, anti-inflammatory, and anti-cancer properties, ${ }^{20,21)}$ is used as a model of a drug with poor water solubility. The loading of curcumin into a PVA filament is easy to measure visually. The color of curcumin depends on its concentration. The yellow color of curcumin changes to orange and black at higher concentration. This unique fluorescence property was used to determine the concentration of curcumin in our previous study. ${ }^{10)}$

In the present study, PVA filaments were incubated in several organic solvents (IPA, $\mathrm{MeOH}, \mathrm{ACE}$, EtOH), in which curcumin was dispersed, at $25^{\circ} \mathrm{C}$ to evaluate curcumin accumulation in PVA filaments over time. The appearance of a curcumin-loaded PVA filament and curcumin content in PVA are shown in Fig. 1. We found that curcumin amount depends on the incubation time for all solvents. The curcumin content in the PVA filament varied with the organic solvent type in the order $\mathrm{MeOH}>\mathrm{EtOH}>\mathrm{ACE}>\mathrm{IPA}$. The PVA filament incubated in $\mathrm{MeOH}$ had the highest curcumin accumulation. This may be due to solubility or organic-solvent-specific swelling property with PVA. We found that $\mathrm{MeOH}$ significantly increased the weight of the PVA filament compared to that obtained with ACE (data not shown). The results suggest that the adsorption of $\mathrm{MeOH}$ led to the swelling of PVA. The interaction of the organic solvent with PVA may have affected curcumin accumulation in the PVA filament.

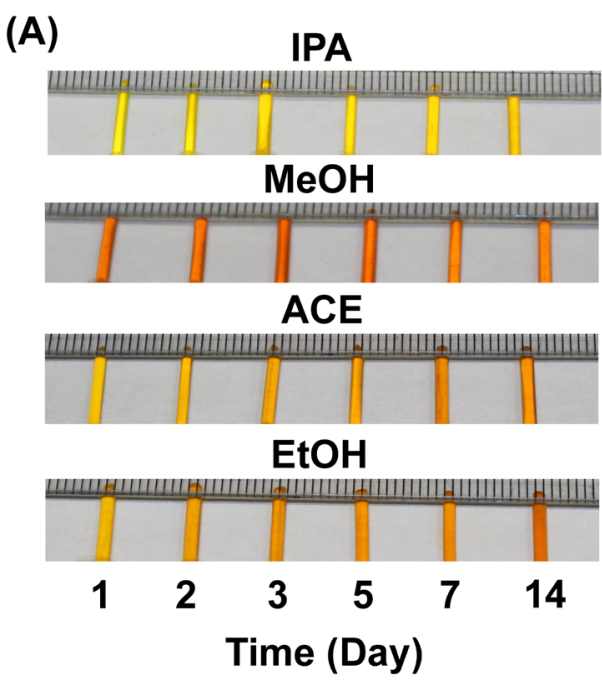

(B)

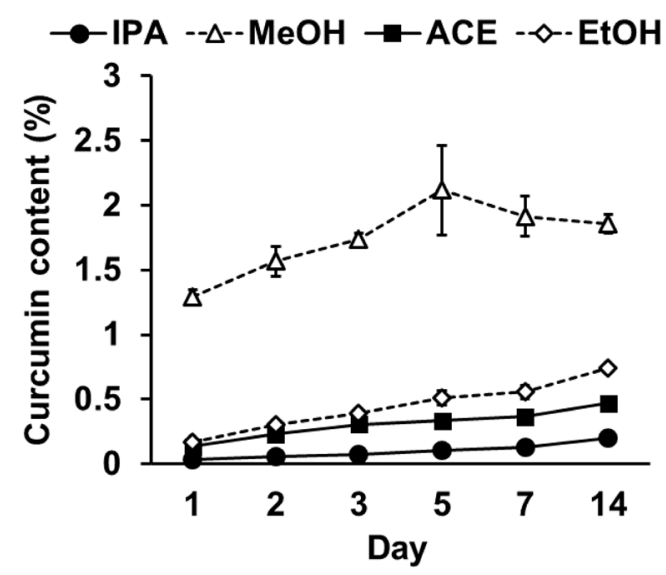

Fig. 1. Influence of Incubation Time on Curcumin Accumulation in PVA Filament for Various Organic Solvents

(A) Appearance of curcumin-loaded PVA filament. (B) Curcumin content in PVA filament over time. Data are the means \pm standard deviation $(n=3)$. Curcumin was dispersed into organic solvents $(10 \mathrm{mg} / \mathrm{mL}$, as $1 \% \mathrm{w} / \mathrm{v}$; undissolved curcumin powder precipitated in the tube). (Color figure can be accessed in the online version.)

Influence of Incubation Temperature on Curcumin Loading into PVA Filament Next, the influence of temperature on curcumin accumulation in the PVA filament was investigated. We assumed that increasing temperature would increase curcumin accumulation in PVA. One reason is that increasing temperature increases the solubility of curcumin in the organic solvent and the higher curcumin concentration may increase infiltration. Another possible reason is that the incubation of PVA filament at around the glass transition temperature of PVA $\left(80^{\circ} \mathrm{C}\right)$ increases curcumin accumulation not only by relaxing the polymer arrangement but also by swelling the polymer filament. The appearance of a curcumin-loaded PVA filament dissolved with an organic solvent at various temperatures is shown in Fig. 2A, and curcumin content in the PVA filament is shown in Fig. 2B. The curcumin amount obtained with heating $\left(80^{\circ} \mathrm{C}\right)$ was much higher than that obtained without heating $\left(25^{\circ} \mathrm{C}\right)$ for all organic solvents (IPA, $\mathrm{MeOH}, \mathrm{ACE}$, and EtOH had 14.5-, 3.0-, 4.8-, and 12.5-fold increases at higher temperature after $4 \mathrm{~h}$ of incubation; see 
(A)

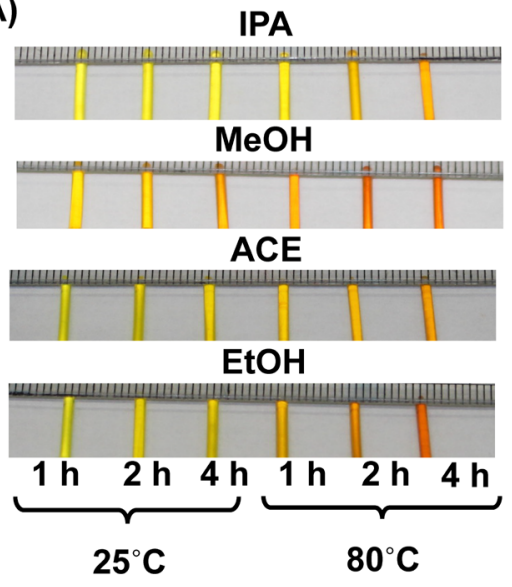

(B)

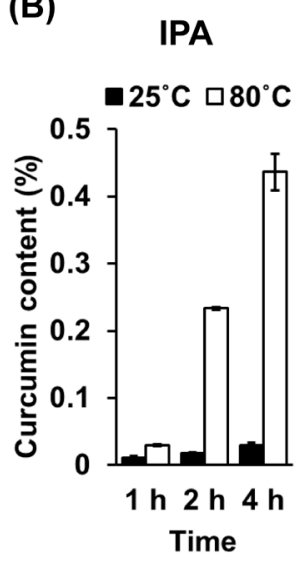

$\mathrm{MeOH}$

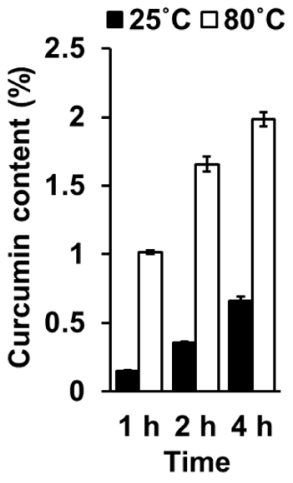

ACE

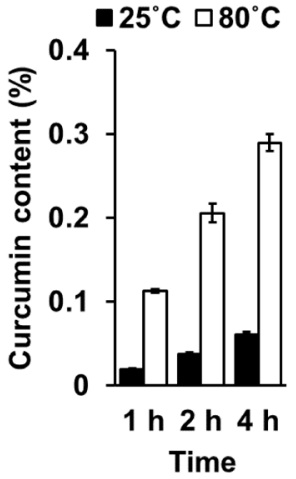

EtOH

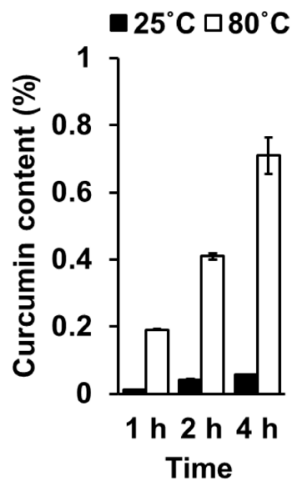

(C)

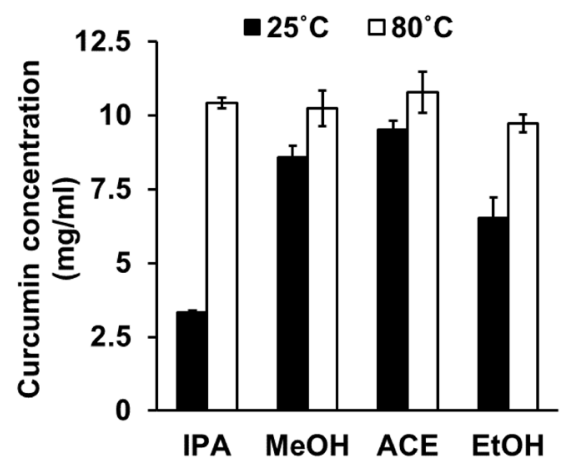

Fig. 2. Influence of Incubation Temperature on Curcumin Accumulation in PVA Filament for Various Organic Solvents

(A) Appearance of curcumin-loaded PVA filament. (B) Curcumin content in PVA filament incubated at 25 and $80^{\circ} \mathrm{C}$. (C) Curcumin concentration in incubation tube after $4 \mathrm{~h}$ of incubation. Data are the means \pm standard deviation $(n=3)$. Curcumin was dispersed into organic solvents $(10 \mathrm{mg} / \mathrm{mL}$, as $1 \% \mathrm{w} / \mathrm{v})$. $($ Color figure can be accessed in the online version.)

Fig. 2B). The curcumin content was found to increase with incubation time in the organic solvents $(1,2$, and $4 \mathrm{~h})$. To reach the curcumin content in the PVA filaments obtained after incubation at $80^{\circ} \mathrm{C}$ for $4 \mathrm{~h}$, much longer incubation was required at $25^{\circ} \mathrm{C}$ (IPA: $0.44 \pm 0.03 \%$ at $80^{\circ} \mathrm{C}$ for $4 \mathrm{~h} v s .0 .20 \pm 0.01 \%$ at $25^{\circ} \mathrm{C}$ for $14 \mathrm{~d}$; $\mathrm{MeOH}$ : $1.98 \pm 0.05 \%$ at $80^{\circ} \mathrm{C}$ for $4 \mathrm{~h} v s$. $2.12 \pm 0.34 \%$ at $25^{\circ} \mathrm{C}$ for $5 \mathrm{~d}$; ACE: $0.29 \pm 0.01 \%$ at $80^{\circ} \mathrm{C}$ for $4 \mathrm{~h}$ vs. $0.30 \pm 0.01 \%$ at $25^{\circ} \mathrm{C}$ for $3 \mathrm{~d}$; $\mathrm{EtOH}: 0.71 \pm 0.06 \%$ at $80^{\circ} \mathrm{C}$ for $4 \mathrm{~h} v s$. $0.75 \pm 0.02 \%$ at $25^{\circ} \mathrm{C}$ for $14 \mathrm{~d}$; see Figs. $2 \mathrm{~B}$ and $1 \mathrm{~B}$ ).

The amount of curcumin solved in the organic solvents at various temperatures was investigated (Fig. 2C). The solu- bility of curcumin in the tube was increased at $80^{\circ} \mathrm{C}$ for all organic solvents. The amount of curcumin dissolved in IPA greatly increased, whereas that dissolved in $\mathrm{MeOH}, \mathrm{ACE}$, and EtOH only slightly increased. The curcumin concentration in organic solvent was almost $10 \mathrm{mg} / \mathrm{mL}$ at $80^{\circ} \mathrm{C}$ (Fig. 2C). These results indicate that curcumin added into the tube was mostly dissolved in the organic solvent $(1 \% \mathrm{w} / \mathrm{v})$. Nevertheless, the curcumin content greatly varied among the organic solvents at $80^{\circ} \mathrm{C}$ (Fig. 2B). Although it is difficult to identify the reason, the solubility of curcumin (curcumin concentration in the solution) may have partially contributed to the increased curcumin accumulation with heating. The order 
of curcumin content in PVA filaments dissolved in different organic solvents is mostly consistent with the results of longterm incubation $(\mathrm{MeOH}>\mathrm{EtOH}>\mathrm{ACE}>\mathrm{IPA}$; see Fig. 1B). These results confirm that the organic solvent type affected curcumin accumulation in the PVA filaments. The interaction among curcumin, organic solvent, and PVA may be important for the incorporation of curcumin into PVA filaments using the soaking method.

Influence of Excess Amount of Curcumin Dispersion on Curcumin Loading into PVA Filament In the present study, an excess amount of curcumin was dispersed in ethanol solution and the curcumin content vs. curcumin ethanolic concentration was investigated, since the use of ethanol is acceptable for the safety and residual solvent in the development of drug formulation. The curcumin added into the tube was partially dissolved in the incubation process with heating; the undissolved curcumin precipitated at the bottom of conical tube, as shown in Fig. 3A. After $4 \mathrm{~h}$ of incubation at $80^{\circ} \mathrm{C}$, the color of the curcumin ethanolic solution drastically changed as the added amount of curcumin was increased (Fig. 3A). The color changed to yellow $(2.0,6.67 \mathrm{mg} / \mathrm{mL})$, orange $(20 \mathrm{mg} / \mathrm{mL})$, brown $(66.7 \mathrm{mg} / \mathrm{mL})$, and almost black $(200,333 \mathrm{mg} / \mathrm{mL})$. The appearance of curcumin-loaded PVA was very different among the samples (Fig. 3B). The curcumin content in the PVA filament remarkably increased as the curcumin concentration increased (Fig. 3C). We found that a maximum of approximately $5 \%$ curcumin was loaded into the PVA filament using the simple soaking method. Of note, the part of the PVA filament that touched the precipitated curcumin (see Fig. 3A) had a darker color (Fig. 3B). We assume that the formation of a diffusion layer of the curcumin solution around the solid curcumin may have created a gradient of drug concentration, and the curcumin molecules diffused into and infiltrated the PVA matrix.

The physical properties of curcumin-loaded PVA filaments were investigated using DSC and XRD analysis. The results are shown in Figs. $4 \mathrm{~A}$ and $\mathrm{B}$, respectively. We found that curcumin-loaded PVA lacked a curcumin peak in both DSC and XRD. No curcumin peak was observed even for the sample treated with the highest concentration of curcumin $(333 \mathrm{mg} / \mathrm{mL}$, sample $\mathrm{h}$ in Figs. 4A and B). PVA may have masked this peak. Although the DSC curve and XRD pattern of the physical mixture of curcumin powder (sample $\mathrm{i}$ in Figs. 4A and B) lacked the curcumin peak, a slightly weak curcumin peak appeared in the magnified XRD pattern (Supplementary Fig. 1). Therefore, we suppose that the curcumin molecules in the PVA filament incorporated using the soaking method are dispersed and present in amorphous form.

Preparation of Curcumin-Loaded PVA Filament Using Synthetic Curcumin To determine the difference between curcumin extract (investigated above) and synthetic curcumin, synthetic-curcumin-loaded PVA was prepared and characterized. It is estimated that curcumin extract contains other curcuminoids; however, details are not known. As shown in Supplementary Fig. 2A, the color of the solution changed from yellow to orange with increasing curcumin concentration. The appearance and curcumin content of synthetic-curcuminloaded PVA filaments are shown in Supplementary Figs. $2 \mathrm{~B}$ and $\mathrm{C}$, respectively. The curcumin content in the PVA filaments increased with increasing curcumin concentration in the solution. The color of the filaments changed from yel-
(A)

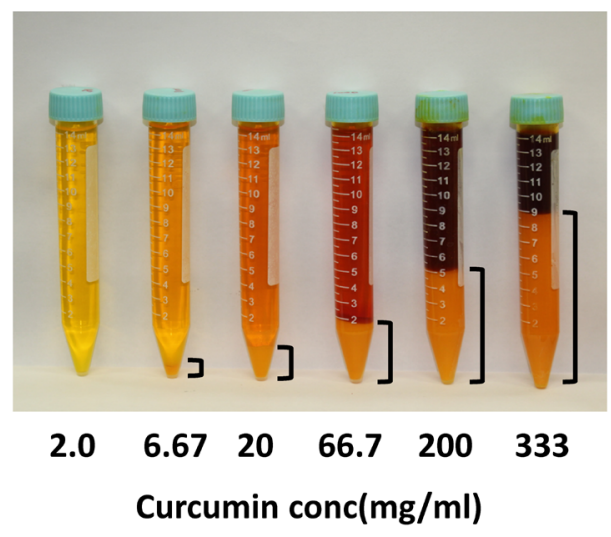

(B)

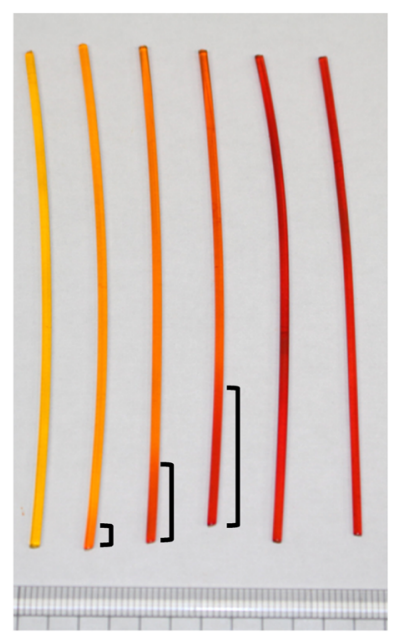

(C)

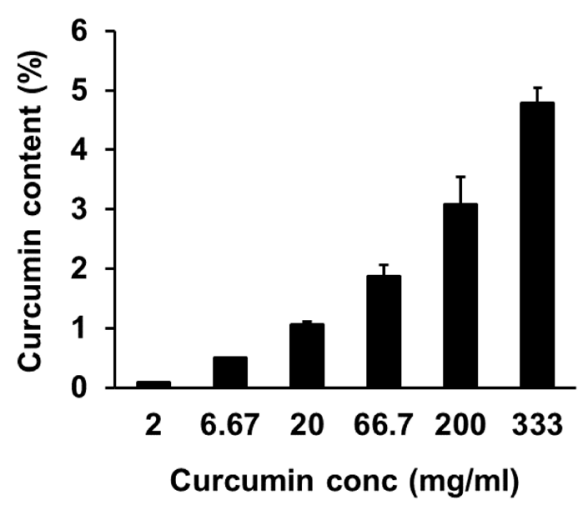

Fig. 3. Influence of Excess Amount of Curcumin Dispersion on Curcumin Content in PVA Filament in Ethanolic Solution after $4 \mathrm{~h}$ of Incubation at $80^{\circ} \mathrm{C}$

(A) Appearance of curcumin dispersion after incubation (curcumin concentration: $2-333 \mathrm{mg} / \mathrm{mL}$ ). The photograph was taken after cooling. (B) Appearance of curcumin-loaded PVA filament. (C) Curcumin content in PVA filament versus curcumin concentration in the incubation tube after $4 \mathrm{~h}$ of incubation. Data are the means \pm standard deviation $(n=3)$. (Color figure can be accessed in the online version.)

low to orange. Approximately $1 \%$ curcumin was incorporated into the PVA filament after incubation in the curcumin dispersion (at $333 \mathrm{mg} / \mathrm{mL}$ ) (Supplementary Fig. 2C). We also found that the part of the synthetic-curcumin-loaded filament that touched the precipitated curcumin became orange (Supple- 
mentary Fig. 2B), as was the case for curcumin extract (Fig. 3B). The incubation of a polymer filament with a solid-phase drug may be a useful strategy for increasing drug content. Other drugs will be investigated in future experiments. The solubility of curcumin extract is much higher than that of synthetic curcumin, as shown in Supplementary Fig. 2D.

\section{(A)}

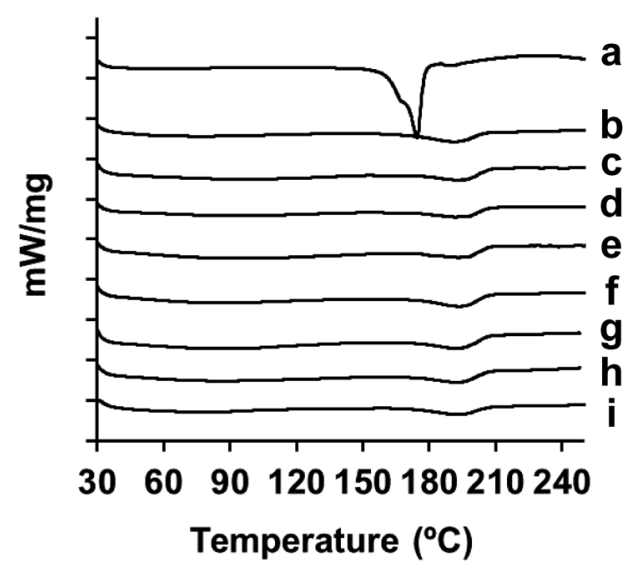

(B)

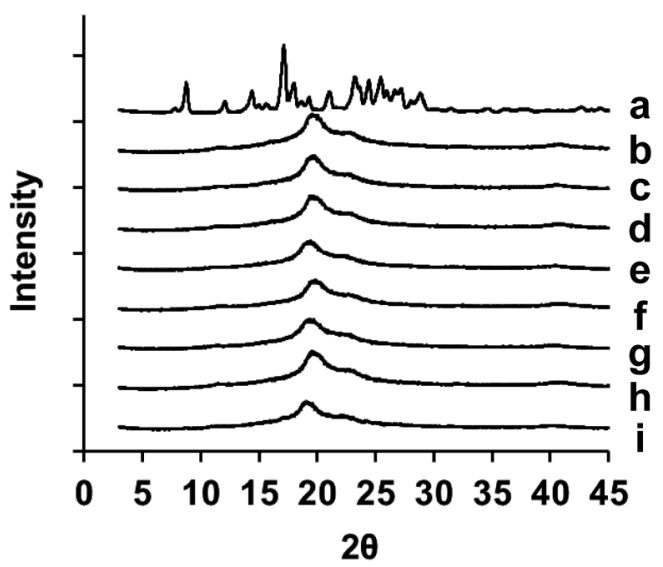

Fig. 4. (A) DSC and (B) XRD Analysis of Pulverized Filament Samples

a. Curcumin powder, b. PVA filament, c. curcumin-loaded PVA filament (incubated in curcumin ethanolic solution at $2 \mathrm{mg} / \mathrm{mL}$ ), d. curcumin-loaded PVA filament $(6.67 \mathrm{mg} / \mathrm{mL})$, e. curcumin-loaded PVA filament $(20 \mathrm{mg} / \mathrm{mL})$, f. curcuminloaded PVA filament $(66.7 \mathrm{mg} / \mathrm{mL})$, g. curcumin-loaded PVA filament $(200 \mathrm{mg} / \mathrm{mL})$, h. curcumin-loaded PVA filament $(333 \mathrm{mg} / \mathrm{mL})$, and i. physical mixture of PVA and curcumin. For the preparation of the physical mixture of curcumin and PVA powder (sample i), 5\% curcumin was included in the sample to compare curcuminloaded PVA samples treated with the highest concentration $(333 \mathrm{mg} / \mathrm{mL})$ (sample h).
These results suggest that the type of curcumin in the solution affects the resulting curcumin content in the PVA filament (i.e., $5 \%$ for curcumin extract $v$ s. $1 \%$ for synthetic curcumin synthetic).

3D Printing Using Curcumin-Loaded PVA Filament and Analysis of Dissolution Profile Curcumin-loaded PVA filaments with various amounts of curcumin extract (Fig. 3) were used to prepare $3 \mathrm{D}$-printed tablets. Typical images of curcumin-loaded PVA tablets are shown in Fig. 5A. We found that brindled tablets were obtained in the experimental condition (Fig. 5A, formulation "333," top view), while curcumin-loaded PVA-based tablets could be prepared by

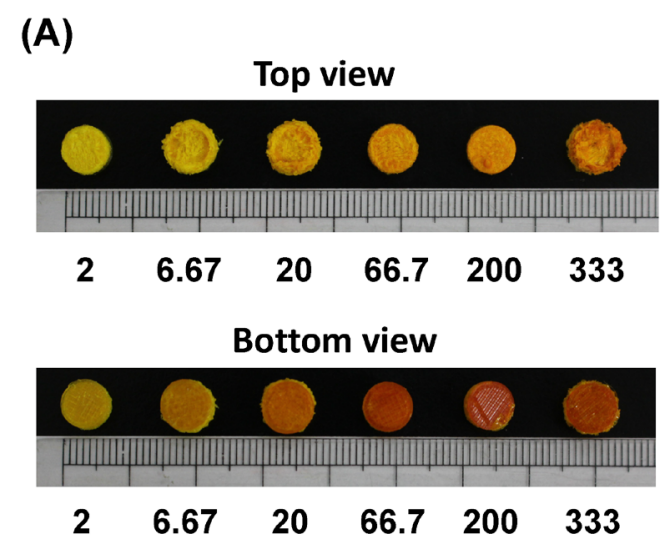

(B)

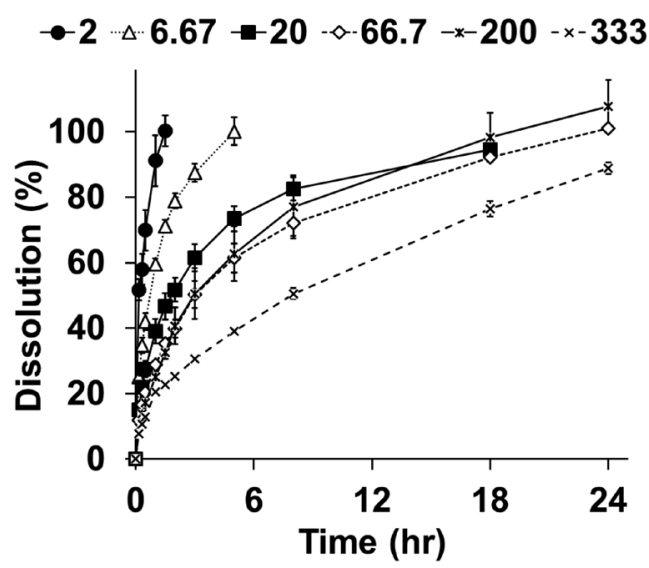

Fig. 5. 3D-Printed PVA Tablets Containing Various Amounts of Curcumin

(A) Top and bottom views of 3D-printed tablets. Curcumin-loaded filaments incubated with $2-333 \mathrm{mg} / \mathrm{mL}$ ethanolic solution were used for the preparation of tablets. (B) Dissolution profiles of curcumin from 3D-printed PVA tablets containing various amounts of curcumin. Data are the means \pm standard deviation $(n=3)$. (Color figure can be accessed in the online version.)

Table 1. Fitting Analysis of Dissolution Curve

\begin{tabular}{|c|c|c|c|c|c|c|}
\hline Formulation & Zero order $\left(r^{2}\right)$ & First order $\left(r^{2}\right)$ & Higuchi $\left(r^{2}\right)$ & \multicolumn{3}{|c|}{ Korsmeyer-Peppas $\left(r^{2}, n\right.$ and mechanism) } \\
\hline 2 & 0.7656 & 0.9779 & 0.9599 & \multicolumn{3}{|c|}{ Not applicable } \\
\hline 6.67 & 0.7811 & 0.9848 & 0.9653 & 0.9997 & 0.48 & non-Fickian transport \\
\hline 20 & 0.6747 & 0.9543 & 0.9137 & 0.997 & 0.5 & non-Fickian transport \\
\hline 66.7 & 0.8213 & 0.9867 & 0.972 & 0.9996 & 0.5 & non-Fickian transport \\
\hline 200 & 0.8367 & 0.9902 & 0.9764 & 0.9969 & 0.5 & non-Fickian transport \\
\hline 333 & 0.9334 & 0.9883 & 0.999 & 0.9956 & 0.48 & non-Fickian transport \\
\hline
\end{tabular}

Data used to obtain the dissolution profiles were obtained from Fig. 5B. The $r^{2}$ value for each model was obtained using Microsoft Excel. 
using FDM-type 3D printer. Although we do not have clear answer about it, we guess that the drying process (remaining ethanol) after the soaking of curcumin may affect the printing of PVA-based tablets. The dissolution of curcumin from the 3D-printed tablets was assessed using the paddle method. As shown in Fig. 5B, there was a tendency for drug dissolution to be delayed as the amount of curcumin incorporated into the tablets increased. For formulation "2", curcumin was released completely at $1.5 \mathrm{~h}$. In contrast, several parts of the tablet with formulation " 333 " were still undissolved at $24 \mathrm{~h}$. These results may be due to the poor water solubility of curcumin. Although PVA is water-soluble, PVA tablets that contained higher amounts of curcumin had delayed dissolution.

The drug dissolution profiles obtained in the present study (Fig. 5B) were analyzed by fitting them to typical drug release models. In this study, zero-order, first-order, Higuchi, and Korsmeyer-Peppas models were used for the fitting of dissolution data. ${ }^{18,22}$ As shown in Table 1, almost all formulations were well-fitted with the first-order, Higuchi or Korsmeyer-Peppas model. For formulation "2", we did not analyze the Korsmeyer-Peppas model because an insufficient number of data points were obtained. The fitting with the Higuchi model suggests that the drug was released from the PVA-based tablets by Fickian diffusion through gel layer. XRD and DSC data suggested that curcumin was present in an amorphous state in the PVA filament. These fitting results confirm that curcumin was relatively homogenously dispersed and infiltrated the PVA filament. Additionally, the fitting with the Korsmeyer-Peppas model indicates that the release profile for 3D-printed tablets obtained from a curcumin-loaded PVA filament can be categorized into anomalous transport that is not completely followed by typical Fick transport. The values of exponent $n$ in the Korsmeyer-Peppas model for the various tablet formulations were similar (approx. 0.5). We suppose that the infiltration of curcumin into the PVA filament may be via the same mechanism for all curcumin concentrations. According to the report about 3D printed hydroxypropyl methyl cellulose-based tablets, the range of $n$ values ( 0.45 to 0.89 ) suggested that the drug release was via diffusion through the hydrated PVA gel layer on the surface of the tablet and PVA polymer erosion. $^{23)}$

\section{CONCLUSION}

This study investigated drug loading into PVA filaments for preparing 3D-printed tablets. We achieved improved drug loading using a simple soaking method. The organic solvent type, temperature, and drug concentration greatly affected the curcumin content. Heating during incubation accelerated curcumin accumulation. Incubation with a high drug concentration increased the drug content in the PVA filament. The preparation of tablets using FDM with a drug-loaded polymer filament will contribute to the progress of personalized therapy, including the novel design of tablets and polypills.

Acknowledgments This research was partly supported by a Grant-in-Aid for Scientific Research from the Japan Society for the Promotion of Science (19K07170).

Conflict of Interest The authors declare no conflict of interest.
Supplementary Materials The online version of this article contains supplementary materials.

\section{REFERENCES}

1) Palo M, Hollander J, Suominen J, Yliruusi J, Sandler N. 3D printed drug delivery devices: perspectives and technical challenges. Expert Rev. Med. Devices, 14, 685-696 (2017).

2) Alam MS, Akhtar A, Ahsan I, Shafiq-Un-Nabi S. Pharmaceutical product development exploiting 3D printing technology: conventional to novel drug delivery system. Curr. Pharm. Des., 24, 5029-5038 (2018)

3) Preis M, Oblom H. 3D-printed drugs for children-are we ready yet? AAPS PharmSciTech, 18, 303-308 (2017).

4) Khaled SA, Alexander MR, Wildman RD, Wallace MJ, Sharpe S, Yoo J, Roberts CJ. 3D extrusion printing of high drug loading immediate release paracetamol tablets. Int. J. Pharm., 538, 223-230 (2018).

5) Afsana JV, Jain V, Haider N, Jain K. 3D printing in personalized drug delivery. Curr. Pharm. Des., 24, 5062-5071 (2018).

6) Arafat B, Qinna N, Cieszynska M, Forbes RT, Alhnan MA. Tailored on demand anti-coagulant dosing: an in vitro and in vivo evaluation of 3D printed purpose-designed oral dosage forms. Eur. J. Pharm. Biopharm., 128, 282-289 (2018).

7) Pietrzak K, Isreb A, Alhnan MA. A flexible-dose dispenser for immediate and extended release 3D printed tablets. Eur. J. Pharm. Biopharm., 96, 380-387 (2015).

8) Goyanes A, Scarpa M, Kamlow M, Gaisford S, Basit AW, Orlu M. Patient acceptability of 3D printed medicines. Int. J. Pharm., 530, 71-78 (2017).

9) Korte C, Quodbach J. 3D-printed network structures as controlledrelease drug delivery systems: dose adjustment, API release analysis and prediction. AAPS PharmSciTech, 19, 3333-3342 (2018).

10) Tagami T, Fukushige K, Ogawa E, Hayashi N, Ozeki T. 3D printing factors important for the fabrication of polyvinylalcohol filamentbased tablets. Biol. Pharm. Bull., 40, 357-364 (2017).

11) Chai $X$, Chai H, Wang $X$, Yang J, Li J, Zhao Y, Cai W, Tao T, Xiang X. Fused deposition modeling (FDM) 3D printed tablets for intragastric floating delivery of domperidone. Sci. Rep., 7, 2829 (2017).

12) Pereira BC, Isreb A, Forbes RT, Dores F, Habashy R, Petit JB, Alhnan MA, Oga EF. 'Temporary plasticiser': a novel solution to fabricate 3D printed patient-centred cardiovascular 'polypill' architectures. Eur. J. Pharm. Biopharm., 135, 94-103 (2019).

13) Kollamaram G, Croker DM, Walker GM, Goyanes A, Basit AW, Gaisford S. Low temperature fused deposition modeling (FDM) 3D printing of thermolabile drugs. Int. J. Pharm., 545, 144-152 (2018).

14) Long J, Gholizadeh H, Lu J, Bunt C, Seyfoddin A. Application of fused deposition modelling (FDM) method of 3D printing in drug delivery. Curr. Pharm. Des., 23, 433-439 (2017).

15) Censi R, Gigliobianco MR, Casadidio C, Di Martino P. Hot melt extrusion: highlighting physicochemical factors to be investigated while designing and optimizing a hot melt extrusion process. Pharmaceutics, 10, 89 (2018).

16) Tan DK, Maniruzzaman M, Nokhodchi A. Advanced pharmaceutical applications of hot-melt extrusion coupled with fused deposition modelling (FDM) 3D printing for personalised drug delivery. Pharmaceutics, 10, 203 (2018).

17) Tagami T, Nagata N, Hayashi N, Ogawa E, Fukushige K, Sakai N, Ozeki T. Defined drug release from 3D-printed composite tablets consisting of drug-loaded polyvinylalcohol and a water-soluble or water-insoluble polymer filler. Int. J. Pharm., 543, 361-367 (2018).

18) Costa P, Sousa Lobo JM. Modeling and comparison of dissolution profiles. Eur. J. Pharm. Sci., 13, 123-133 (2001).

19) Singhvi G, Songh M. Review: In vitro drug release characterization models. Int. J. Pharm. Sci. Res., 2, 77-84 (2011). 
20) Kunnumakkara AB, Bordoloi D, Harsha C, Banik K, Gupta SC, Aggarwal BB. Curcumin mediates anticancer effects by modulating multiple cell signaling pathways. Clin. Sci. (Lond.), 131, 1781-1799 (2017).

21) Vallianou NG, Evangelopoulos A, Schizas N, Kazazis C. Potential anticancer properties and mechanisms of action of curcumin. Anticancer Res., 35, 645-651 (2015).
22) Dash S, Murthy PN, Nath L, Chowdhury P. Kinetic modeling on drug release from controlled drug delivery systems. Acta Pol. Pharm., 67, 217-223 (2010).

23) Li Q, Guan X, Cui M, Zhu Z, Chen K, Wen H, Jia D, Hou J, Xu W, Yang X, Pan W. Preparation and investigation of novel gastrofloating tablets with 3D extrusion-based printing. Int. J. Pharm., 535, 325-332 (2018). 